\title{
CONTROLO DA FADIGA ASSOCIADA À DOENCA ONCOLÓGICA ATRAVÉS DE PROGRAMAS DE EXERCICIO FÍSICO: UMA SCOPING REVIEW
}

\author{
Catarina Rodrigues \\ Instituto de Ciências Biomédicas Abel Salazar/ UP (Estudante); Oncologia/CHTMAD \\ acataryn@gmail.com \\ Bárbara Gomes \\ Escola Superior de Enfermagem do Porto; UNIESEP/CINTESIS \\ bgomes@esenf.pt \\ Carlos Albuquerque \\ Escola Superior de Saúde de Viseu - IPV, Viseu, Portugal \\ Health Sciences Research Unit: Nursing (UICISA: E) \\ cmalbuquerque@gmail.com
}

Recepción Artículo: 27 agosto 2021 Admisión Evaluación: 07 septiembre 2021 Informe Evaluador 1: 12 septiembre 2021 Informe Evaluador 2: 13 septiembre 2021 Aprobación Publicación: 15 septiembre 2021

\begin{abstract}
RESUMO
Objectivo: Determinar a efetividade das intervenções de enfermagem baseadas no exercício na redução da fadiga associada à doença oncológica. Métodos: Realizada uma scoping review, recorrendo às bases de dados PubMed, CINHAL complete, BVS, Scielo e ao motor de busca google académico. Os estudos eram incluídos se fossem publicados nos últimos 10 anos, em português, inglês ou espanhol e que se referissem a intervenções baseadas no exercício, com impacto na fadiga associada à doença oncológica, implementadas exclusivamente por enfermeiros. 0 corpus ficou constituído por 11 artigos. Resultados: Os resultados obtidos sugerem que 0 exercício, durante ou após os tratamentos oncológicos, pode reduzir os efeitos da fadiga na pessoa com diferentes diagnósticos de cancro. Os vários programas de exercício testados incluem o treino aeróbio, de resistência e/ou estiramento muscular, que diferem na sua duração, frequência, intensidade e grau de supervisão. Conclusões: Os enfermeiros podem desenvolver a sua prática na educação da pessoa com doença oncológica para os benéficos do exercício no controlo da fadiga, mas podem ainda desenvolver e supervisionar programas baseados no exercício, integrados numa equipa multidisciplinar, de forma a obterem os melhores resultados possíveis para esta população específica.
\end{abstract}

Palavras-chave: fadiga; cancro; exercício; reabilitação

\section{ABSTRACT}

Controlling fatigue associated with cancer disease through exercise programs: a scoping review. Objective: To determine the effectiveness of exercise-based nursing interventions in reducing fatigue associated with cancer. Methods: A scoping review was carried out, using the PubMed, CINHAL complete, BVS, 


\section{CONTROLO DA FADIGA ASSOCIADA À DOENGA ONCOLÓGICA ATRAVÉS DE PROGRAMAS DE EXERCÍCIO FÍSICO: UMA SCOPING REVIEW}

Scielo databases and the academic google search engine. Studies were included if they were published in the last 10 years, in Portuguese, English or Spanish, and if they referred to exercise-based interventions, with an impact on fatigue associated with cancer, implemented exclusively by nurses. The corpus consisted of 11 articles.

Results: The results obtained suggest that exercise, during or after cancer treatments, can reduce the effects of fatigue in people with different cancer diagnoses. The various exercise programs tested include aerobic, resistance and/or muscle stretch training, which differ in their duration, frequency, intensity and degree of supervision. Conclusions: Nurses can develop their practice in educating people with cancer about the benefits of exercise in controlling fatigue, but they can also develop and supervise exercise-based programs, as part of a multidisciplinary team, in order to obtain the best possible results. for this specific population.

Keywords: fatigue; cancer; exercise; rehabilitation

\section{INTRODUÇÃO}

Um diagnóstico de cancro pode tornar-se um momento de aprendizagem, uma vez que a pessoa está mais disponível para mudar o seu comportamento. Porém, as pessoas tendem a tornar-se menos ativas durante 0 tratamento da doença oncológica, podendo até ficar mesmo inativas. Os profissionais de saúde possuem então uma oportunidade para intervir nesta fase em que a pessoa esta mais recetiva à aprendizagem, e proceder a uma avaliação das suas necessidades, disponibilizar suporte motivacional e uma multiplicidade de exercícios, respeitando as suas preferências e necessidades. Desta forma, é fornecido um suporte para que as mudanças permaneçam a longo prazo, mesmo para as pessoas que estejam na fase de tratamento ativo ou com doença oncológica avançada (Foster et al., 2019).

Á medida que o número de casos de cancro aumenta, também se assiste a um incremento da fadiga associada à doença oncológica. Desta forma, os enfermeiros e as associações profissionais de enfermagem têm a possibilidade de defender políticas de saúde para a sua comunidade. Os enfermeiros estão associados aos avanços ocorridos na educação para a saúde das comunidades e à promoção das mudanças ocorridas nas políticas de saúde, sendo estas intervenções de enfermagem reconhecidas como imprescindíveis para melhorar a saúde das populações (Djalilova et al., 2019).

Apesar dos avanços ocorridos no tratamento do cancro, ainda são observadas morbilidades significativas relacionadas com o mesmo. A fadiga associada ao cancro (FAC) pode ser uma consequência da doença oncológica, bem como dos efeitos secundários resultantes do tratamento (Scott \& Posmontier, 2017). É considerada um problema importante de saúde pública (Djalilova et al., 2019), sendo o efeito adverso mais frequente nos doentes oncológicos, afetando-os em cerca de 75\% a 100\% dos casos, durante ou após o diagnóstico de cancro, e pode persistir frequentemente durante meses ou anos (National Cancer Institute, 2021). A FAC é definida pelo National Comprehensive Cancer Network (2021), como uma sensação angustiante, persistente e subjetiva de cansaço ou exaustão física, emocional e/ou cognitiva, relacionada ao cancro ou ao seu tratamento, que não é proporcional à atividade recente e interfere no funcionamento normal da pessoa. Os sobreviventes referem complicações relacionadas à fadiga, quer de ordem física, quer psicossocial, que podem reduzir a qualidade de vida associada à saúde (Scott \& Posmontier, 2017).

A adoção de um estilo de vida onde se inclua o exercício, acarreta muitos benefícios para a saúde e o mesmo acontece na pessoa com doença oncológica. Os resultados de vários estudos demonstram que as intervenções baseadas no exercício, durante ou após os tratamentos da doença oncológica, além de melhorar o bem-estar físico, podem ajudar na gestão dos efeitos adversos associados aos tratamentos, onde se inclui a FAC, e impactar significativamente a qualidade de vida das pessoas com diferentes diagnósticos de cancro (Scott \& Posmontier, 2017; Segal et al., 2017).

0 exercício físico é considerado seguro, quer durante o tratamento ativo, quer após o término do mesmo e deve fazer parte do continuum de tratamento de todos os indivíduos com cancro (Foster et al., 2019; Segal et al., 2017). Diversas organizações internacionais publicaram recomendações para 0 exercício para pessoas com doença oncológica ou sobreviventes de cancro, como por exemplo a American Cancer Society, o American 
College of Sports Medicine (ACSM), o Exercise and Sports Science Australia, o Cancer Care Ontario e a Clinical Oncology Society of Austrália. De acordo com peritos da ACSM, existe evidência científica suficiente para sustentar a eficácia de esquemas específicos de treino físico, de forma a gerir os resultados em saúde associados ao cancro, onde se inclui a FAC, a qualidade de vida, o funcionamento físico, a ansiedade e os sintomas depressivos (Campbell et al., 2019).

A evidência científica sustenta uma abordagem vigorosa multifacetada ( $85 \%$ a $95 \%$ da frequência cardíaca máxima) e/ou de intensidade leve (50\% a 70\% da frequência cardíaca máxima) no que se refere à atividade aeróbia, treino de resistência, treino de relaxamento e massagem, por forma a reduzir a fadiga em doentes oncológicos (McGowan, 2016). Assim, pode permitir-se que a pessoa determine o tipo de exercício que prefere implementar para o treino aeróbio e de resistência, pois os benefícios são semelhantes (Segal et al., 2017).

\section{OBJETIVOS DO ESTUDO}

0 propósito desta Scoping Review é determinar em que medida as intervenções de enfermagem baseadas no exercício físico, podem controlar a fadiga associada à doença oncológica.

\section{METODOLOGIA}

Nesta Scoping Review foram considerados os estudos que incluíssem enfermeiros com especialidade em oncologia ou em reabilitação ou que, independentemente da sua especialização, prestassem cuidados de enfermagem baseados em evidência, com intervenções específicas fundamentadas em programas de exercício físico com 0 intuito de reduzir os sintomas de FAC. Seguimos as diretrizes do Joanna Briggs Institute Reviewer's Manual, de forma a identificar, selecionar e avaliar criteriosamente a literatura relevante. A estratégia de pesquisa assentou em três etapas, com o intuito de identificar os estudos primários e revisões publicados e não publicados. Inicialmente, foi realizada uma pesquisa nas bases de dados PubMed e CINHAL, seguida de uma análise das palavras usadas no título e resumo, e dos termos indexados utilizados para descrever os artigos encontrados. Á posteriori foi realizada uma segunda análise, recorrendo às palavras-chave e termos indexados encontrados, em todas as bases de dados previamente selecionadas. Por último, a partir da lista de referências dos artigos incluídos nesta revisão, pesquisámos estudos adicionais.

Os artigos publicados em inglês, espanhol ou português foram considerados para inclusão. 0 período selecionado para pesquisa foi desde janeiro de 2009 até dezembro de 2019, uma vez que os artigos publicados por enfermeiros no que respeita a intervenções baseadas no exercício físico para controlo da fadiga associada à doença oncológica são escassos. As bases de dados selecionadas foram: PubMed (US National Library of Medicine National Institutes of Health), CINAHL Complete (Cumulative Index to Nursing and Allied Health Literature), SciELO (Scientific Electronic Library Online) e BVS (biblioteca virtual em saúde). A pesquisa por literature cinzenta (grey literature) incluiu o motor de busca Google Scholar.

Esta revisão incluiu diferentes tipos de estudos, nomeadamente meta-analises, revisões integrativas ou sistemáticas, ensaios clínicos randomizados (ECR), observacionais, entre outros. Os dados incluídos nos artigos e necessários para análise, foram retirados por dois revisores distintos, com recurso a uma ferramenta adaptada para extração de dados do JBI (JBI Manual for Evidence Synthesis, 2019). Os dados colhidos dos estudos incluem informações estandardizadas (autor, ano da publicação, país), desenho do estudo, detalhes sobre as intervenções de enfermagem (contexto), duração da intervenção, população-alvo, tamanho da amostra e os principais resultados.

Os resultados desta revisão são apresentados sob a forma de tabelas. Estes são acompanhados de um texto sumário narrativo, que pretende descrever em que medida os resultados estão relacionados com os objetivos e a questão norteadora da scoping review. A avaliação da qualidade e do risco de viés dos estudos incluídos não foi realizada, uma vez que o objetivo desta revisão não é obter respostas para uma pergunta específica, mas fornecer uma visão geral da evidência científica existente sobre as diversas intervenções de enfermagem baseadas no exercício físico para controlo da FAC. 


\section{RESULTADOS}

Através da estratégia de pesquisa realizada nas bases de dados selecionadas, obtivemos um total de 557 estudos. Para além destes, foram identificados mais 19 artigos, conseguidos através de outras fontes. Após se terem eliminado os documentos em duplicado, os títulos e resumos de 565 artigos foram estudados e desses, 177 foram considerados para uma análise detalhada do texto integral. Um total de 166 estudos com texto integral foram excluídos, uma vez que não cumpriam os critérios de inclusão, resultando num total de 11 artigos incluídos para revisão. De seguida, é apresentado um fluxograma detalhado com o número de estudos incluídos em cada uma das etapas (Figura 1)

Figura 1. Fluxograma referente à seleção dos estudos e ao processo de inclusão
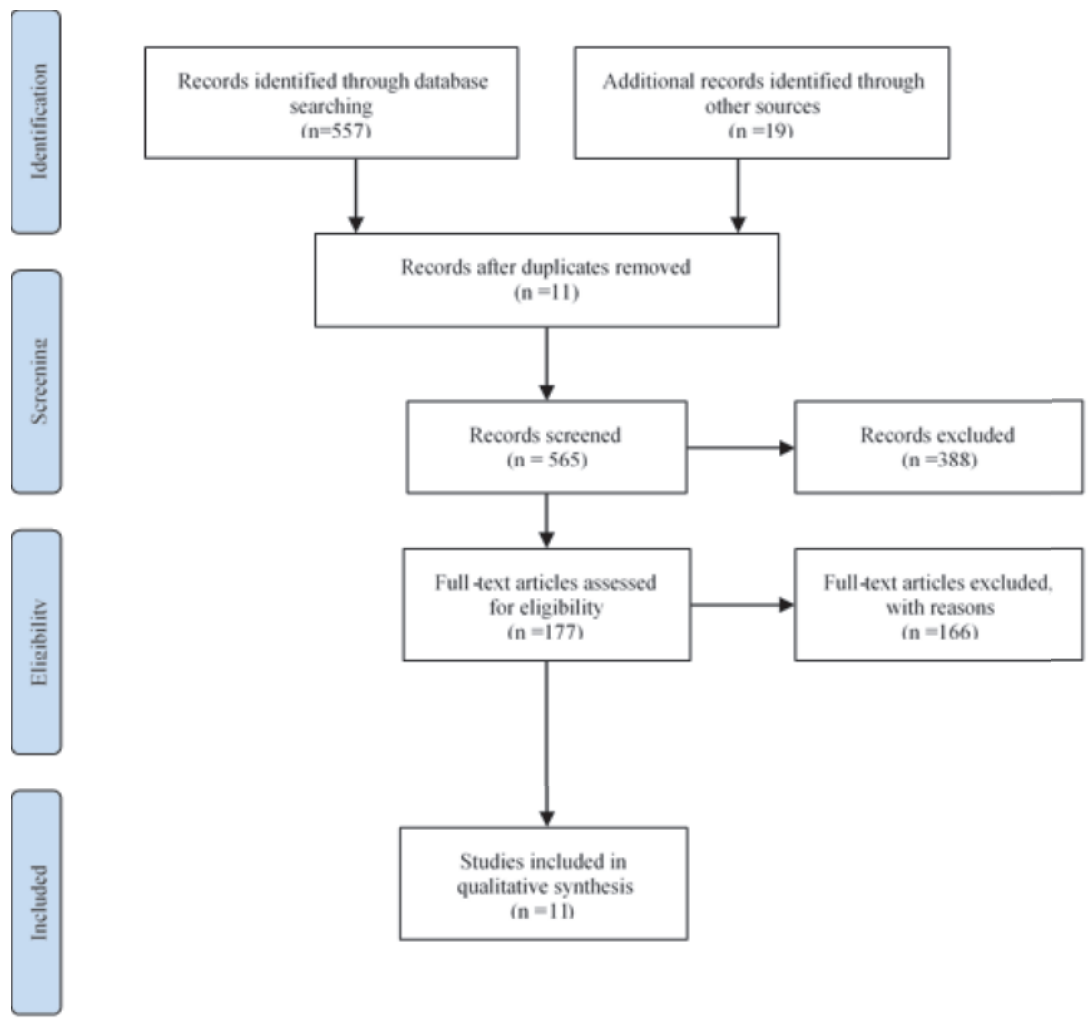

From: Moher D, Liberati A, Tetzlaff J, Altman DG, The PRISMA Group (2009). Preferred Reporting Atems for Systematic Reviews and Meta-Analyses: The PRISMA Statement. PLoS Med 6(7): e1000097. doi:10.1371/journal.pmed1000097

Os resultados obtidos nos diferentes estudos sugerem que as intervenções de enfermagem baseadas no exercício físico, durante ou após o término dos tratamentos oncológicos, podem ter um impacto significativo no controlo da fadiga em indivíduos com diferentes diagnósticos de cancro.

Esta revisão apresenta intervenções fundamentadas no exercício com o principal intuito de reduzir a FAC. Contudo, verifica-se que estas intervenções também podem ter um impacto positivo noutros sintomas ou desafios, analisados de forma adicional, como nas perturbações do sono, na dor, depressão, dispneia, dependência para as atividades de vida diária (AVD), aptidão para o exercício e autoeficácia, e na qualidade de vida (QV). 
Os artigos incluídos nesta revisão foram publicados entre os anos de 2011 e 2018, e foram desenvolvidos maioritariamente nos Estados Unidos da América $(n=6)$. Os restantes estudos foram conduzidos na Colômbia, Coreia do Sul, Dinamarca, Reino Unido e em Taiwan. Nesta revisão foram incluídos artigos com diferentes desenhos de estudo: ECR $(n=2)$, experimental $(n=1)$, pré-teste/pós-teste $(n=2)$, revisão integrativa ou sistemática $(n=4)$, meta-análise $(n=1)$ e descritivo transversal $(n=1)$.

A maioria dos estudos não especifica a localização tumoral, ou referem-se a diferentes tipos de cancro ( $n=8)$. Porém, verifica-se que dois artigos referem-se apenas a mulheres com o diagnóstico de cancro de mama, enquanto que outros estudos compreendem este diagnóstico, entre outros tipos de cancro $(n=4)$. Apenas um artigo foi desenvolvido em indivíduos com tumores de cabeça e pescoço.

Todos os estudos incluídos nesta revisão foram conduzidos em adultos com doença oncológica em diferentes momentos da sua trajetória de tratamento. A maioria das intervenções de enfermagem baseadas no exercício foram implementadas na fase de tratamento ativo de quimio e/ou radioterapia ou transplante medular $(n=5)$, ou na fase de sobrevivência $(n=5)$. Apenas um estudo se refere a pessoas com doença oncológica em fase paliativa. Os restantes artigos não especificam em que fase do tratamento do cancro, as intervenções foram executadas ( $n=3$ ).

As intervenções baseadas no exercício físico e implementadas por enfermeiros, com o intuito de controlar a FAC, foram analisadas em diferentes estudos. Adamsen et al. (2012),desenvolveram um programa de exercício multimodal supervisionado ("The Body \& Cancer Trial"), que compreendia um treino cardiovascular de alta intensidade e de exercícios de alta resistência, acoplado com técnicas de relaxamento, treino de consciência corporal e massagem. Apuraram que a fadiga reduziu significativamente no grupo de intervenção, apesar da heterogeneidade dos doentes oncológicos, no que respeita ao diagnóstico e ao esquema de quimioterapia instituído.

Numa meta-análise realizada acerca da gravidade da FAC e que incluiu 113 ECRs com intervenções fundamentadas no exercício, psicológicas, e a associação do exercício com intervenções psicológicas, verificou-se uma melhoria nos sintomas de fadiga durante e após o tratamento inicial. Porém, estes estudos apresentam limitações uma vez que a maioria foi realizada em mulheres com cancro de mama ou em sobreviventes de neoplasia da mama. Concluíram que alguns modos de intervenção específica são mais efetivos no tratamento da FAC em diferentes momentos da trajetória do tratamento do cancro (Mustian et al., 2017).

Foram incluídos para estudo quatro artigos de revisão baseados no exercício, combinado com outras intervenções, que demonstraram que 0 exercício produz um efeito paliativo nos sintomas de fadiga durante 0 tratamento ativo, mas que após a conclusão dos tratamentos oncológicos, traduz-se em reabilitação (Egan et al., 2013; Mitchell et al., 2015), o que incrementa a qualidade de vida global (Scott \& Posmontier, 2017). Além do que foi referido, verificou-se que a atividade física supervisionada e que 0 treino resistido melhoram significativamente, não apenas a FAC, mas também os sintomas de depressão e 0 bem-estar físico e a funcionalidade (Meneses-Echavez et al., 2014).

Para além dos estudos já referidos anteriormente, e que possuem o seu foco na melhoria da fadiga, existem outros artigos que também demonstraram um efeito benéfico neste sintoma, recorrendo a programas de exercício físico ( Huether et al., 2016; McDonald et al., 2018). Os programas de caminhada incluídos nesta revisão, estão maioritariamente focados em controlar, não apenas a FAC, mas também as perturbações do sono e a dor (Boehmke et al., 2011; Egan et al., 2013). Outros estudos possuem também como objetivo incrementar a mobilidade, a atividade física e/ou a adesão ao exercício físico (Roh et al., 2014; Shang et al., 2013).

Os estudos analisados evidenciaram programas de exercício físico, implementados por enfermeiros com 0 intuito de controlar a FAC, com algumas diferenças (conteúdo, frequência, duração, intensidade e grau de supervisão), nomeadamente quando acoplados a outro tipo de intervenções. Desta forma, torna-se difícil avaliar os benefícios apenas do exercício (Tabela 1). 
Tabela 1. Intervenções baseados no exercício para controlo da FAC

\begin{tabular}{|c|c|c|c|c|}
\hline Autor/País & $\begin{array}{l}\text { Desenho do } \\
\text { estudo }\end{array}$ & Intervenção & População & Resultados \\
\hline $\begin{array}{l}\text { Huether et al, } \\
\text { 2016/EUA }\end{array}$ & $\begin{array}{l}\text { Pré/Pós } \\
\text { teste }\end{array}$ & $\begin{array}{l}\text { Energy Through Motion: sessões } \\
\text { regulares com os participantes } \\
\text { (fornecido KIT ETM) para apoiar o } \\
\text { incremento e/ou manutenção da } \\
\text { atividade física de intensidade baixa } \\
\text { a moderada e/ou exercícios de } \\
\text { resistência. Reforço via contacto } \\
\text { telefónico ( } 3 \text { meses) }\end{array}$ & $\begin{array}{l}50 \quad \text { doentes } \\
\text { oncológicos em } \\
\text { tratamento ou } \\
\text { follow-up: } \\
\text { grupo } \\
\text { cuidados } \\
\text { habituais }(\mathrm{n}=30) \\
\text { e grupo ETM } \\
(\mathrm{n}=20\end{array}$ & $\begin{array}{l}\text { Participantes do grupo } \\
\text { ETM apresentaram } \\
\text { incrementos nos níveis de } \\
\text { atividade física e da } \\
\text { qualidade de vida (QV) e } \\
\text { redução da fadiga }\end{array}$ \\
\hline $\begin{array}{l}\text { Boehmke et al, } \\
\text { 2011/ Taiwan }\end{array}$ & $\begin{array}{l}\text { Experiment } \\
\text { al } \quad(2 \\
\text { grupos })\end{array}$ & $\begin{array}{l}\begin{array}{l}\text { Programa de } 6 \\
\text { caminhada em semanas de } \\
\text { regime de }\end{array} \\
\text { ambulatório (após a cirurgia, } 3 \text { a } 5 \\
\text { sessões por semana e pelo menos } 30 \\
\text { min por sessão) associado a um } \\
\text { plano estratégico para aumentar a } \\
\text { auto-eficácia do exercício }\end{array}$ & $\begin{array}{l}72 \text { mulheres } \\
\text { com ca. Mama } \\
\text { (estadio I/II) a } \\
\text { aguardar } \\
\text { quimioterapia } \\
\text { (QT): grupo } \\
\text { controlo ( } \mathrm{n}=37 \text { ) } \\
\text { e grupo de } \\
\text { intervenção } \\
(\mathrm{n}=35)\end{array}$ & $\begin{array}{l}\text { Participantes do grupo } \\
\text { intervenção apresentaram } \\
\text { melhores índices de QV, } \\
\text { menos fadiga e } \\
\text { perturbações do sono, } \\
\text { melhor auto-eficácia, } \\
\text { capacidade e aptidão para o } \\
\text { exercício }\end{array}$ \\
\hline $\begin{array}{l}\text { McDonald et } \\
\text { al., 2018/EUA }\end{array}$ & $\begin{array}{l}\text { Pré/Pós } \\
\text { teste } \\
\text { grupo) }\end{array}$ & $\begin{array}{l}\text { Intervenção personalizada de } 6 \\
\text { semanas baseada no comportamento } \\
\text { associado à atividade física com } \\
\text { recurso a exergames (PAfitME): } \\
\text { Wii Fit exergames, com visita } \\
\text { domiciliária de enfermeira (1h) e } \\
\text { contacto telefónico semanal (10 } \\
\text { min) durante } 3 \text { semanas após as } 6 \\
\text { semanas de visita domicliária (9 } \\
\text { semanas) }\end{array}$ & $\begin{array}{lr}8 & \text { indivíduos } \\
\text { com CCP na } \\
\text { fase } & \text { pós } \\
\text { tratamento }\end{array}$ & $\begin{array}{l}\text { A fadiga reduziu de forma } \\
\text { significativa, bem como a } \\
\text { dependência para as AVD. } \\
\text { Aumento da capacidade de } \\
\text { equilíbrio, } \\
\text { cardiorrespiratória, força } \\
\text { muscular, flexão do ombro. }\end{array}$ \\
\hline $\begin{array}{l}\text { Mitchell et al, } \\
2015 / \text { EUA }\end{array}$ & $\begin{array}{l}\text { Revisão } \\
\text { Integrativa }\end{array}$ & $\begin{array}{l}\text { Exercício/atividade } \\
\text { (caminhar, física } \\
\text { exercícios de resistência ou } \\
\text { exercícios combinados), que } \\
\text { diferem na duração, frequência, } \\
\text { intensidade e grau de supervisão; } \\
\text { reabilitação } \\
\text { estruturada multidimensional } \\
\text { intervenção (treino físico, } \\
\text { aconselhamento psicoeducativa, } \\
\text { massagem, relaxamento e drenagem } \\
\text { manual linfática); tratamento de } \\
\text { sintomas concomitantes (dor, } \\
\text { insónia, depressão, dispneia) }\end{array}$ & $\begin{array}{l}\text { Participantes } \\
\text { com ca. da } \\
\text { mama, cólon ou } \\
\text { próstata, } \\
\text { submetidos a } \\
\text { radio e/ou QT } \\
\text { ou transplante } \\
\text { medular, e } \\
\text { sobreviventes } \\
\text { de cancros } \\
\text { sólidos ou } \\
\text { hemato- } \\
\text { oncológicos }\end{array}$ & $\begin{array}{l}\text { Intervenções recomendadas } \\
\text { para controlo da FAC: } \\
\text { exercício, reabilitação } \\
\text { estruturada, controlo de } \\
\text { sintomas e avaliação de } \\
\text { fatores de risco. O } \\
\text { exercício produz um efeito } \\
\text { paliativo na fadiga durante } \\
\text { os tratamentos e após os } \\
\text { mesmos traduz-se em } \\
\text { reabilitação. }\end{array}$ \\
\hline $\begin{array}{l}\text { Egan et al, } 2013 \\
\text { / Reino Unido }\end{array}$ & $\begin{array}{l}\text { Revisão } \\
\text { Sistemática }\end{array}$ & $\begin{array}{l}\text { Reabilitação na fase da } \\
\text { sobrevivênvia: exercício e/ou } \\
\text { reabilitação física (tai-chi, } \\
\text { incremento da funcionalidade dos } \\
\text { membros superiores, exercício } \\
\text { estruturado, caminhada, exercícios } \\
\text { aeróbicos e de resistência na fase } \\
\text { pós tratamento), massagem, treino } \\
\text { da musculatura pélvica, exercício } \\
\text { supervisionado para a depressão } \\
\text { (sessões superiores a } 30 \text { min) }\end{array}$ & $\begin{array}{l}\text { Mulheres com } \\
\text { ca. mama ou } \\
\text { ginecológico; } \\
\text { pessoas com } \\
\text { cancro do cólon } \\
\text { ou pâncreas; } \\
\text { sobreviventes } \\
\text { com múltipla } \\
\text { natureza de } \\
\text { neoplasia }\end{array}$ & $\begin{array}{l}\text { O exercício/reabilitação } \\
\text { motora demonstraram } \\
\text { efetividade na redução da } \\
\text { fadiga após os tratamentos } \\
\text { na maioria dos } \\
\text { diagnósticos de cancro }\end{array}$ \\
\hline $\begin{array}{l}\text { Mustian et al, } \\
2017 / \text { EUA }\end{array}$ & Metanálise & $\begin{array}{l}\text { Exercício (aeróbio, anaeróbio ou de } \\
\text { força, ou ambos), intervenção } \\
\text { psicológica, a combinação dos } \\
\text { anteriores e intervenções } \\
\text { farmacológicas para controlo da } \\
\text { FAC) }\end{array}$ & $\begin{array}{l}11525 \\
\text { participantes: } \\
\text { mulheres com } \\
\text { cancro da mama } \\
(46,9 \%) \text { e os } \\
\text { restantes com } \\
\text { diferentes tipos } \\
\text { de cancro }\end{array}$ & $\begin{array}{l}\text { Incluídos } 113 \text { ECR. O } \\
\text { exercício, intervenções do } \\
\text { foro psicológico e a } \\
\text { combinação de ambos, } \\
\text { melhoraram a FAC durante } \\
\text { e após o tratamento, mas as } \\
\text { intervenções } \\
\text { farmacológicas não tiveram } \\
\text { impacto, }\end{array}$ \\
\hline
\end{tabular}




\begin{tabular}{|c|c|c|c|c|}
\hline $\begin{array}{l}\text { Andamsen et al, } \\
2013 / \\
\text { Dinamarca }\end{array}$ & ECR & $\begin{array}{l}\text { The Body \& Cancer Trial: } \\
\text { Intervenção multifacetada baseada } \\
\text { no exercício supervisionado, treino } \\
\text { cardiovascular de alta-intensidade e } \\
\text { de alta resistência ( } 10 \text { min em } \\
\text { bicicleta estática, com uma } \\
\text { intensidade de } 85-95 \% \text { da FC } \\
\text { máxima), relaxamento e treino de } \\
\text { consciência do corpo } \\
\text { (alongamentos, respiração, Yoga e } \\
\text { Pilatos) e massagem, } 9 \mathrm{~h} / \mathrm{semana} \\
\text { durante } 6 \text { semanas }\end{array}$ & $\begin{array}{l}213 \text { indivíduos } \\
\text { com diferentes } \\
\text { diagnósticos de } \\
\text { cancro e } \\
\text { submetidos a } \\
\text { quimioterapia: } \\
\text { grupo controlo } \\
(\mathrm{n}=107) \text { e grupo } \\
\text { de intervenção } \\
(\mathrm{n}=106)\end{array}$ & $\begin{array}{lr}\text { FAC } & \text { reduziu } \\
\text { significativamente } & \text { no } \\
\text { grupo de intervenção } & \end{array}$ \\
\hline $\begin{array}{l}\text { Shang et al, } \\
2012 / \text { EUA }\end{array}$ & ECR & $\begin{array}{l}\text { Análise secundária de um programa } \\
\text { de exercícios de caminhada e } \\
\text { fortalecimento muscular leve a } \\
\text { moderado, em ambulatório: } \\
\text { caminhada de } 10 \mathrm{~min} \text {, aumentando } \\
\text { para } 30 \mathrm{~min} \text {, durante } 5 \text { dias por } \\
\text { semana; contacto telefónico } \\
\text { quinzenal. Programa realizado entre } \\
5 \text { a } 15 \text { semanas }\end{array}$ & $\begin{array}{l}126 \text { indivíduos } \\
\text { com ca da } \\
\text { mama } \\
\text { próstata ou } \\
\text { tratamento } \quad(\mathrm{QT} \\
\text { e/ou RT): grupo } \\
\text { controlo }(\mathrm{n}=58) \\
\text { e grupo de } \\
\text { exercício } \\
(\mathrm{n}=68)\end{array}$ & $\begin{array}{l}\text { Taxa de adesão ao } \\
\text { exercício no GI foi } 32,35 \% \\
(4 \text { sessões e } 113 \\
\text { min/semana). Este grupo } \\
\text { manteve ou aumentou os } \\
\text { seus níveis de exercício, } \\
\text { reduzindo a fadiga. A } \\
\text { conservação de energia é } \\
\text { uma estratégia usada no } \\
\text { controlo da fadiga }\end{array}$ \\
\hline $\begin{array}{l}\text { Roh et al, } 2014 \text { / } \\
\text { Coreia }\end{array}$ & $\begin{array}{l}\text { Descritivo } \\
\text { transversal }\end{array}$ & $\begin{array}{l}\text { Avaliação baseada no desempenho: } \\
6 \text { min de marcha; entrevista semi- } \\
\text { estruturada ( } 6 \text { meses) }\end{array}$ & $\begin{array}{l}91 \text { doentes } \\
\text { oncológicos } \\
\text { idosos em } \\
\text { cuidados } \\
\text { paliativos }\end{array}$ & $\begin{array}{l}\text { A distância média dos } 6 \\
\text { MM foi } 220,38 \mathrm{~m} \text {. Altos } \\
\text { níveis de mobilidade estão } \\
\text { correlacionados com níveis } \\
\text { baixos de fadiga e dor e } \\
\text { perturbações do sono }\end{array}$ \\
\hline $\begin{array}{l}\text { Meneses- } \\
\text { Echavez et al, } \\
2014 \\
\text { Colombia }\end{array}$ & $\begin{array}{l}\text { Revisão } \\
\text { sistemática }\end{array}$ & $\begin{array}{l}\text { Atividade física supervisionada: } \\
\text { aeróbica (caminhada, bicicleta } \\
\text { estática), treino de resistência e/ou } \\
\text { programas de alongamento } \\
\text { muscular }\end{array}$ & $\begin{array}{l}\text { Doentes } \\
\text { oncológicos } \\
\text { com diferentes } \\
\text { tratamentos ou } \\
\text { estadio da } \\
\text { doença } \\
\end{array}$ & $\begin{array}{l}14 \text { ECR incluídos. A } \\
\text { atividade física } \\
\text { supervisionada melhora } \\
\text { significativamente a FAC. } \\
\text { O mesmo foi conseguido } \\
\text { com o treino de resistência }\end{array}$ \\
\hline $\begin{array}{l}\text { Scott \& } \\
\text { Posmontier } \\
2017 / \text { EUA }\end{array}$ & $\begin{array}{l}\text { Revisão } \\
\text { Integrativa }\end{array}$ & $\begin{array}{l}\text { Exercícios aeróbios ou de } \\
\text { resistência, de fortalecimento } \\
\text { muscular, treino de flexibilidade, } \\
\text { com ou sem supervisão, com } \\
\text { duração, frequência semanal e } \\
\text { intensidades distintas e podem estar } \\
\text { associados a apoio psicossocial. } \\
\text { Como complemento os programas } \\
\text { podem incluir exercícios de } \\
\text { relaxamento, Yoga básico ou } \\
\text { música e variam entre } 6 \text { semanas a } \\
6 \text { meses de duração }\end{array}$ & $\begin{array}{l}\text { Doentes } \\
\text { oncológicos } \\
\text { durante ou após } \\
\text { a fase ativa de } \\
\text { tratamento, com } \\
\text { diferentes tipos } \\
\text { de diagnóstico }\end{array}$ & $\begin{array}{l}7 \text { estudos incluídos. O } \\
\text { exercício reduz os efeitos } \\
\text { da FAC e aumenta a QV. } \\
\text { Os enfermeiros podem } \\
\text { desenvolver programas } \\
\text { para controlo da fadiga } \\
\text { durante e após os } \\
\text { tratamentos oncológicos }\end{array}$ \\
\hline
\end{tabular}

\section{DISCUSSÃO}

Nas equipas de saúde, os enfermeiros que prestam cuidados a pessoas com doença oncológica, estão preparados para garantir que os indivíduos em risco de deficiência, limitação de atividade e de restrições na participação são identificados, avaliados e ainda, que serão providenciados cuidados imprescindíveis no domínio da reabilitação, com 0 objetivo de incrementar a função e a qualidade de vida. Desta forma, estes profissionais podem agir no sentido de integrar a reabilitação na trajetória de tratamento do cancro (Campbell \& Reigle, 2020), possibilitando também o controlo de sintomas e desafios associadas à doença oncológica e/ou ao seu tratamento, como a FAC. 


\section{CONTROLO DA FADIGA ASSOCIADA À DOENGA ONCOLÓGICA ATRAVÉS DE PROGRAMAS DE EXERCÍCIO FÍSICO: UMA SCOPING REVIEW}

As intervenções baseadas no exercício são cada vez mais reconhecidas como um elemento imprescindível do tratamento e dos cuidados de suporte dos sobreviventes de cancro (Batalik, et al., 2021). Os estudos incluídos na revisão compreendiam maioritariamente os exercícios aeróbios e/ou o treino resistido. Porém, a frequência, a intensidade, a duração e 0 modo de implementação divergiu entre as diferentes intervenções apresentadas. Verificou-se, mesmo assim, uma melhoria nos sintomas da FAC.

A investigação realizada no domínio da fadiga em doentes oncológicos, incluiu frequentemente a referência a medidas de fadiga auto-reportada, e os dados que se referem a correlatos biológicos ou fisiológicos são escassos (National Cancer Institute, 2021), o que também foi observado nos estudos incluídos nesta revisão.

Alguns artigos analisados, não estavam apenas focados no controlo da fadiga, mas também na melhoria de outros sintomas associados ao cancro e no incremento da qualidade de vida. De acordo com Alfano \& Pergolotti (2018), a dose e o tipo de intervenção específica de reabilitação na doença oncológica necessitam de ser personalizados, tendo em consideração a informação clínica, fisiológica, psicossocial e os dados fornecidos pela pessoa, respeitando se o seu desejo é restaurar a função, incrementar a participação, e/ou prevenir os efeitos tardios dos tratamentos oncológicos.

Nalguns dos estudos incluídos, as intervenções baseadas no exercício foram implementadas em mulheres com o diagnóstico de cancro de mama, ou em pessoas que além deste diagnóstico, possuem outros tipos de cancro. Apesar do desenvolvimento da prática clínica e do conhecimento obtido com estes trabalhos, são necessários estudos adicionais que incluem pessoas com outros tipos de cancro, nomeadamente doenças hemato-oncológicas, pois cada diagnóstico e tratamento podem impactar de forma distinta cada indivíduo. Também é necessário realizar investigação complementar em doentes oncológicos com doença avançada ou paliativa, pois como verificámos nesta scoping review, apenas um estudo explorou de forma isolada esta etapa da trajetória do cancro.

Apesar das limitações inerentes a esta revisão, este documento pode servir como uma base para os enfermeiros que prestam cuidados a pessoas com doença oncológica e tenham a intenção de desenvolver programas de exercício para controlar a FAC. Uma das limitações é que alguns dos estudos incluídos possuam amostras reduzidas ou são pequenos estudos piloto. Além disso, as ferramentas ou escalas de avaliação da fadiga também diferem entre pesquisadores, tornando difícil estabelecer conclusões baseadas em evidência, acerca das intervenções analisadas. A formação dos profissionais de saúde que desempenham funções na área da oncologia deve evoluir tendo em consideração a evidência científica e as diretrizes para 0 exercício, para que a sua implementação seja bem-sucedida. Os profissionais de enfermagem precisam de um grande conhecimento acerca dos benefícios e segurança do exercício, de forma a aconselhar os doentes oncológicos durante e após os tratamentos instituídos (Stout et al., 2020).

\section{CONCLUSÃO}

Os enfermeiros possuem competências que Ihes permitem intervir junto da pessoa com doença oncológica, não apenas na educação sobre os benefícios do exercício físico, mas também para desenvolver e supervisionar programas baseados no exercício, integrados numa equipa multidisciplinar, de forma a obter os melhores resultados possíveis para os indivíduos com cancro e fadiga associada. Os efeitos positivos destas intervenções já são reconhecidos. Contudo, é necessário realizar mais investigação que permita desenvolver programas de exercício com intervenções específicas individualizadas, para diferentes diagnósticos e etapas específicas de tratamento do cancro.

\section{REFERÊNCIAS BIBLIOGRÁFICAS}

Adamsen, L., Ejlertsen, B., Midtgaard, J., Stage, M., Bloomquist, K., Rørth, M., Andersen, C., Møller, T., \& Quist, M. (2012). The effects of a six-week supervised multimodal exercise intervention during chemotherapy on cancer-related fatigue. European Journal of Oncology Nursing, 17(3): 331-339.

https://doi.org/10.1016/j.ejon.2012.09.003 
Alfano, C. M., \& Pergolotti, M. (2018). Next-Generation Cancer Rehabilitation. Rehabilitation Nursing, 43(4): 186-194. https://doi.org/10.1097/rnj.0000000000000174

Batalik, L.; Winnige, P.; Dosbaba, F.; Vlazna, D.; Janikova, A. (2021). Home-Based Aerobic and Resistance Exercise Interventions in Cancer Patients and Survivors: A Systematic Review. Cancers 13 (8), 1915. https://doi.org/10.3390/cancers13081915

Boehmke, M., Dickerson, S. S., Fisher, N., Wu, Y.-W. B., \& Wang, Y.-J. (2011). Effects of a 6-Week Walking Program on Taiwanese Women Newly Diagnosed With Early-Stage Breast Cancer. Cancer Nursing, 34(2), E1-E13. https://doi.org/10.1097/ncc.0b013e3181e4588d

Campbell, G., \& Reigle, B. S. (2020). Introduction: The Case for Cancer Rehabilitation. Seminars in Oncology Nursing, 36(1), 150973. https://doi.org/10.1016/j.soncn.2019.150973

Campbell KL, Winters-Stone K, Wiskemann J, et al. (2019). Exercise guidelines consensus state-for cancer survivors: Consensus statment from international multidisciplinary roundtable. Med Sci Sports Exerc. https://doi:10.1249/MSS.0000000000002116

Djalilova, D., Cramer, M. E., \& Msn, N. M. (2019). Policy analysis of access to and reimbursement for nonpharmacologic therapies for cancer related fatigue. March, 1-6. https://doi.org/10.1111/phn.12615

Egan, M. Y., McEwen, S., Sikora, L., Chasen, M., Fitch, M., \& Eldred, S. (2013). Rehabilitation following cancer treatment. Disability and Rehabilitation, 35(26): 2245-2258.

https://doi.org/10.3109/09638288.2013.774441

Foster, J., Worbey, S., Chamberlain, K., Horlock, R., \& Marsh, T. (2019). Integrating physical activity into cancer care Evidence and guidance. https://www.macmillan.org.uk/assets/integrating-physical-activity-into-cancercare-evidence-and-guidance.pdf

Hauken, M. A., Holsen, I., Fismen, E., \& Bogsnes Larsen, T. M. (2015). Working toward a good life as a cancer survivor: A longitudinal study on positive health outcomes of a rehabilitation program for young adult cancer survivors. Cancer Nursing, 38(1), 3-15. https://doi.org/10.1097/NCC.0000000000000138

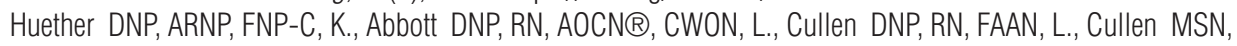
ARNP, ANP-C, GNP-C, L., \& Gaarde RN, BSN, OCN@, A. (2016). Energy Through Motion@: An EvidenceBased Exercise Program to Reduce Cancer-Related Fatigue and Improve Quality of Life. Clinical Journal of Oncology Nursing, 20(3), E60-E70. https://doi.org/http://dx.doi.org/10.1188/16.CJON.E60-E70

Aromataris E, Munn Z (Editors) (2020). JBI Manual for Evidence Synthesis. https://synthesismanual.jbi.global

McDonald, S., Wang, H.-L., Padhya, T., Visovsky, C., Vondruska, K., Huang, L.-T., McMillan, S. C., Huang, Y., Buck, H. G., Russell, J., Vijayakumar, N., \& Gwede, C. (2018). A Behavioral Physical Activity Intervention to Manage Moderate and Severe Fatigue Among Head and Neck Cancer Patients-Pre-efficacy Study in the National Institutes of Health ORBIT Model. Cancer Nursing, OO(0), 1. https://doi.org/10.1097/ncc.0000000000000568

McGowan, K. (2016). Physical exercise and cancer-related fatigue in hospitalized patients: Role of the clinical nurse leader in implementation of interventions. Clinical Journal of Oncology Nursing, 20(1): E20-E27. https://doi.org/10.1188/16.CJON.E20-E27

Meneses-Echavez, J. F., González-Jiménez, E., Correa, J. E., \& Ramírez-Vélez, R. (2014). [Supervised physical activity interventions in the management of cancer-related fatigue: a systematic review]. Nutrición Hospitalaria, 30(3): 486-497. https://doi.org/10.3305/nh.2014.30.3.7635

Mitchell, S. A., Hoffman, A. J., Clark, J. C., DeGennaro, R. M., Poirier, P., Robinson, C. B., \& Weisbrod, B. L. (2014). Putting evidence into practice: an update of evidence-based interventions for cancer-related fatigue during and following treatment. Clinical journal of oncology nursing, 18 Suppl, 38-58. https://doi.org/10.1188/14.CJON.S3.38-58 
Moher D, Liberati A, Tetzlaff J, Altman DG, The PRISMA Group (2009). Preferred Reporting Items for Systematic Reviews and Meta-Analyses: The PRISMA Statement. PLoS Med 6(7): e1000097. https://doi:10.1371/journal.pmed1000097

Mustian KM1, Alfano CM2, Heckler C1, Kleckner AS1, Kleckner IR1, Leach CR2, Mohr D3, Palesh OG4, Peppone LJ1, Piper BF5, Scarpato J6, Smith T2, Sprod LK7, M. S. (2017). JAMA Oncol. 4(11): 961-968. https://doi.org/10.1016/S2214-109X(16)30265-0.Cost-effectiveness

National Cancer Institute (2019). Fatigue - health professional version. https://www.cancer.gov/aboutcancer/treatment/side-effects/fatigue/fatigue-hp-pdq\#cit/section_1.2

National Comprehensive Cancer Network (2021) NCCN Clinical Practice Guidelines in Oncology: Cancer-Related Fatigue. Version 1.2021. Plymouth Meeting, Pa: National Comprehensive Cancer Network. https://www.nccn.org/guidelines/category_1

Nicole L. Stout, Justin C. Brown, Anna L. Schwartz, Timothy F. Marshall, Anna M. Campbell (2020). An Exercise Oncology Clinical Pathway: Screening and Referral for Personalized Interventions. Cancer. 126(12): 27502758. https://doi:10.1002/cncr.32860

Roh, S. Y., Yeom, H. A., Lee, M. A., \& Hwang, I. Y. (2014). Mobility of older palliative care patients with advanced cancer: A Korean study. European Journal of Oncology Nursing, 18(6): 613-618. https://doi.org/10.1016/j.ejon.2014.06.005

Scott, K., \& Posmontier, B. (2017). Exercise interventions to reduce cancer-related fatigue and improve healthrelated quality of life in cancer patients. Holistic Nursing Practice, 31(2): 66-79. https://doi.org/10.1097/HNP.0000000000000194

Segal, R., Zwaal, C., Green, E., Tomasone Phd, J. R., Loblaw Md Msc, A., \& Petrella, T. (2017). EXERCISE FOR PEOPLE WITH CANCER, Segal et al. e290 Exercise for people with cancer: a systematic review. Current Oncology, 24(4): e290. https://doi.org/10.3747/c0.24.3619

Shang, J., Wenzel, J., Krumm, S., Griffith, K., Stewart, K., \& Ed, D. (2013). Who Will Drop Out \& Who Will Drop In, Exercise Adherence in a RCT among Patients Receiving Active Cancer Treatment. 35(4): 312-322. https://doi.org/10.1097/NCC.0b013e318236a3b3.Who 